Document downloaded from:

http://hdl.handle.net/10251/65400

This paper must be cited as:

Guild, M.; Garcia Chocano, VM.; Kan, W.; Sánchez-Dehesa Moreno-Cid, J. (2015). Acoustic metamaterial absorbers based on multilayered sonic crystals. Journal of Applied Physics. 117(11):114902-1-114902-14. doi:10.1063/1.4915346.

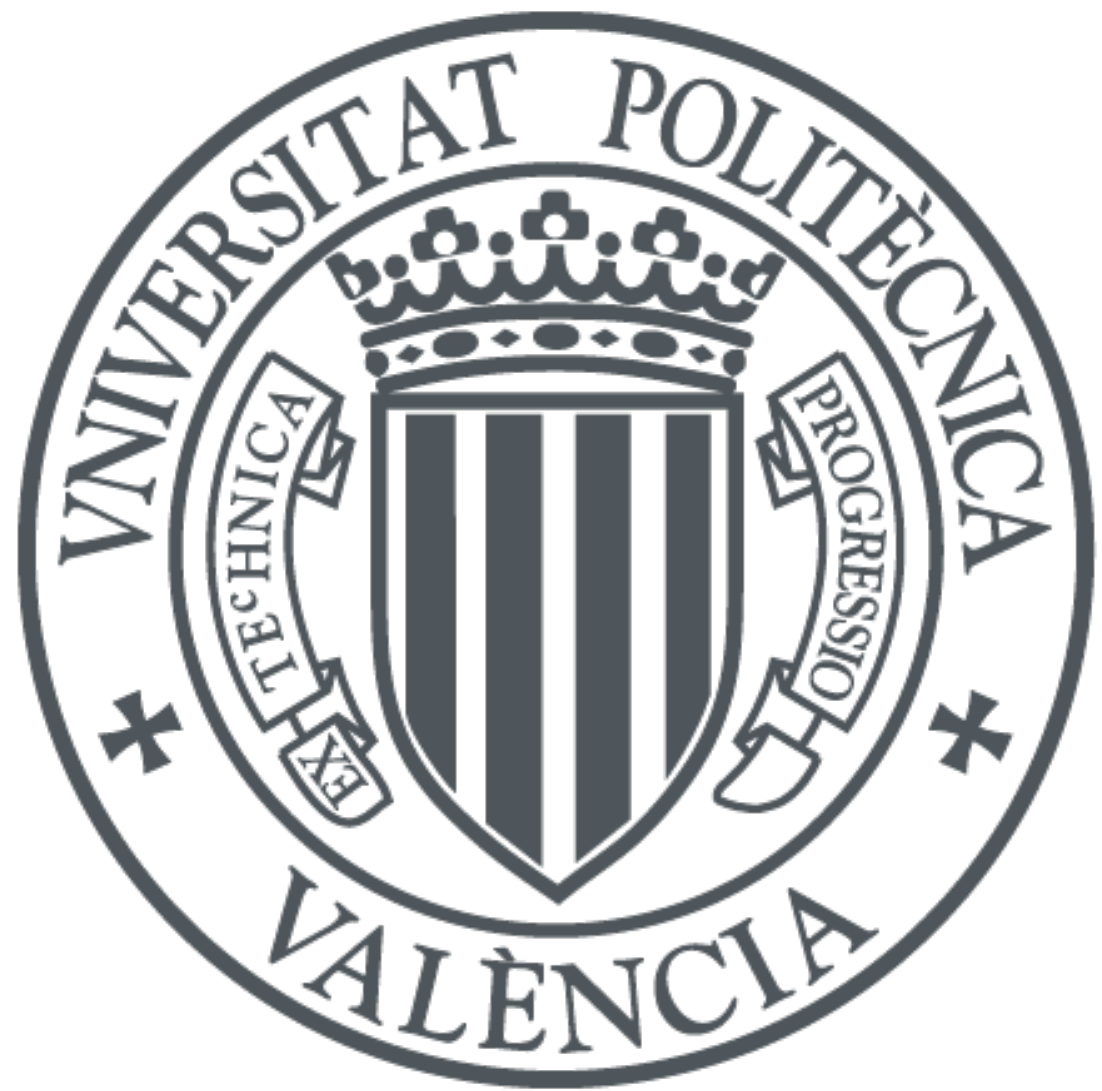

The final publication is available at

http://dx.doi.org/10.1063/1.4915346

Copyright American Institute of Physics (AIP)

Additional Information 


\title{
Acoustic metamaterial absorbers based on multilayered sonic crystals
}

\author{
Matthew D. Guild, ${ }^{1, \text { a) }}$ Victor M. Garcia-Chocano, ${ }^{1}$ Weiwei Kan, ${ }^{2}$ and José Sánchez-Dehesa ${ }^{1, b)}$ \\ ${ }^{1)}$ Grupo de Fenómenos Ondulatorios, Departamento de Ingenieria Electrónica, Universitat Politècnica de València, \\ Camino de vera s $n$, E-46022 Valencia, Spain \\ ${ }^{2)}$ Department of Physics, Key Laboratory of Modern Acoustics, MOE, Institute of Acoustics, Nanjing University, Nanjing 210093, \\ People's Republic of China
}

(Dated: 16 December 2014)

Through the use of a layered arrangement, it is shown that lossy sonic crystals can be arranged to create a structure with extreme acoustic properties, namely an acoustic metamaterial. This artificial structure shows di erent e ective fluid and absorptive properties in di erent orientations. Theoretical, numerical and experimental results examining thermoviscous losses in sonic crystals are presented, enabling the fabrication and characterization of an acoustic metamaterial absorber with complex-valued anisotropic inertia. To accurately describe and fabricate such an acoustic metamaterial in a realizable experimental configuration, confining structures are needed which modify the e ective properties, due to the thermal and viscous boundary layer e ects within the sonic crystal lattice. Theoretical formulations are presented which describe the e ects of these confined sonic crystals, both individually and as part of an acoustic metamaterial structure. Experimental demonstrations are also reported using an acoustic impedance tube. The formulations developed can be written with no unknown or empirical coe cients, due to the structured lattice of the sonic crystals and organized layering scheme, and it is shown that higher filling fraction arrangements can be used to provide a large enhancement in the loss factor.

\section{INTRODUCTION}

Sonic crystals, defined as periodic distributions of sound scatterers in a fluid or air background, have been proposed as structures for attenuating and filtering sound waves because of their acoustic bandgaps ${ }^{1-3}$. Their refractive properties, which were studied in the pioneering work of Kock and Harvey ${ }^{4}$ back in 1949, were later revisited and expanded by Cervera and coworkers 5 . These authors developed an acoustic lens for airborne sound by using a cluster of rigid rods with external lenticular shape. The lensing behavior was understood to result from the e ective properties of the cluster that, at low frequencies, behaves like a homogeneous fluid with some given e ective mass density and bulk modulus. In fact, it has been demonstrated that sonic crystals, with hexagonal and square symmetries, behaves like isotropic fluids whose e ective parameters simply depend on the lattice filling fraction ${ }^{6}$.

Research on sonic crystals below the homogenization limit has been boosted in recent years due to the possibility of using them as artificial structures with extreme homogenized properties, referred to as acoustic metamaterials, behaving as broadband anisotropic fluids, or metafluids ${ }^{7}$. Moreover, acoustic metamaterials or metafluids with mass anisotropy are receiving increasing attention due to the extraordinary acoustic devices predicted from transformation acoustics, like acoustic cloaks and acoustic hyperlenses, which require anisotropic fluids as the principal ingredient ${ }^{8-10}$. Several designs and a few experimental demonstrations of acoustic metamaterials with dynamical mass anisotropy have been reported in the last few years ${ }^{9,11-15}$, which make use of a nonresonant microstructure to create the desired anisotropy.

\footnotetext{
a)Electronic mail: mdguild@utexas.edu; Current address: NRC Postdoctoral Research Associateship Program, U.S. Naval Research Laboratory, Washington DC 20375

b)Electronic mail: jsdehesa@upv.es
}

In most applications, acoustic metamaterials have been envisioned using ideal materials, with the presence of losses seen as a hinderance to the design. However, acoustic waves in fluids such as air or water have inherent losses which arise from thermal and viscous e ects, and can be particularly pronounced for small structures such as those encountered in metamaterial applications. Furthermore, for sound absorber applications, these losses can be significant, and are in fact necessary to achieving the goal of absorbing the acoustic energy. A recent study analyzed the homogenized properties of periodically distributed elastic cylinders embedded in a viscous fluid ${ }^{16}$, however the analysis was constrained by the condition of low filling fractions, where the sound absorbing effects are not significant unless the frequencies are very high, or the structures are very small.

Recently, there has been interest in using the losses within an acoustic metamaterial to provide an enhancement in the absorption, using resonant structures such as membranes and mass-spring-damper systems ${ }^{17-23}$. However, such resonant absorption mechanisms are inherently narrowband, and thus there is a need for nonresonant high loss structures in achieving broadband acoustic metamaterial absorbers. Broadband sound absorption has been recently demonstrated by layered structures of porous materials ${ }^{24}$. Two dimensional sonic crystals made of cylindrical absorbing units were previously employed to fabricate acoustic barriers with broadband sound absorption; their insertion loss being the result of two complementary e ects ${ }^{25,26}$ : i) the absorbing properties of the individual units and ii) the reflectance properties of the periodic structure. Moreover, sonic crystals consisting of rigid rods arranged in a hexagonal lattice with a large filling fraction have been recently employed to dissipate broadband acoustic energy at the core of an omni-directional sound absorber, also known as an acoustic black hole ${ }^{27}$, though the authors did not examine the physical mechanisms of the observed lossy behavior.

In this work, the use of lossy sonic crystals with high fill- 
ing fractions will be examined to demonstrate its applicability for sound absorbers, and how acoustic metamaterials with complex-valued e ective material properties can be created and implemented, allowing for anisotropy in both the sound absorption characteristics and the e ective properties. Through the use of a structured sonic crystal lattice, precise layered arrangements and high filling fractions can be achieved which enable higher losses and large anisotropy in the e ective properties of the resulting acoustic metafluid. Unlike resonant acoustic metamaterial absorbers, these enhanced losses and anisotropy in the absorption characteristic are broadband, due to the non-resonant, sub-wavelength scale of the sonic crystal structure.

The work performed here is described as follows. In Section II, the theoretical formulations and parametric characterization of two-dimensional (2D) sonic crystals with thermovisous losses are presented and verified with numerical simulations. Modifications to these formulations for sonic crystals in a confined test structure are developed in Section III. The properties of these lossy sonic crystals in a complexvalued anisotropic acoustic metafluid are then formulated in Section IV and the experimental results are described in Section V. Finally, Section VI gives a brief sumary of our findins.

\section{TWO-DIMENSIONAL SONIC CRYSTALS}

For thermoviscous fluids, the properties of the sonic crystal are dependent on the size of the thermal and viscous boundary layers relative to that of the cylinder and lattice dimensions. In particular, an expression for the e ective homogenized properties is sought for a lattice of cylinders which are non-interacting, both fluid dynamically (i.e. boundary layers which do not touch) and acoustically (neglecting multiple scattering e ects). Extensive work has been performed on the topic of porous media, and detailed models have been developed to describe such systems. The specific formulations in each case depend on the configuration of the microstructure. Two-dimensional sonic crystals, which consist of parallel cylinders in a structured lattice, represent an idealized arrangement of a fibrous porous media, and previous work on such fibrous porous materials can provide a basis for development of a model for lossy sonic crystals. A theoretical formulation for 2D lossy sonic crystals is presented in Section II A, from which a nondimensional parameter space is developed and discussed in Section II B. The theoretical results are then compared and verified with Comsol Multiphysics simulations in Section II C.

\section{A. Theoretical formulation for $2 \mathrm{D}$ sonic crystals}

The general form of the bulk density for rigid fibrous media consisting of parallel cylinders, such as the configuration illustrated in Fig. 1, can be expressed as ${ }^{28}$

$$
\text { e } 0 \frac{-}{1 f}
$$

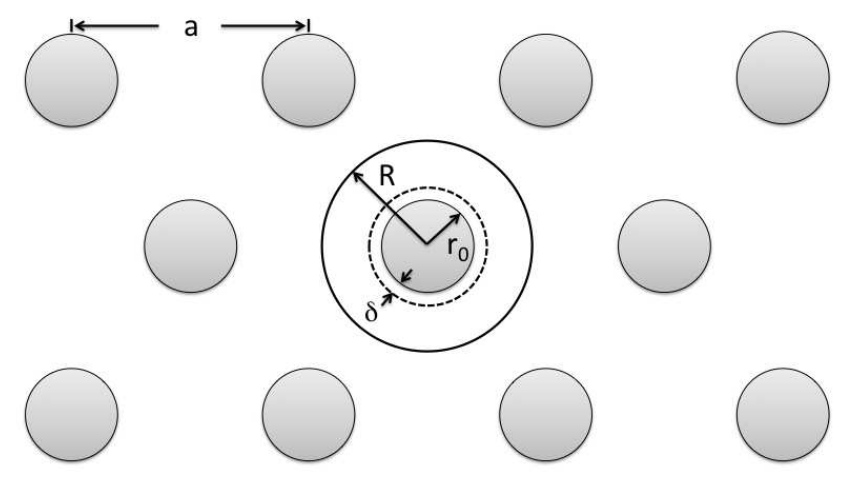

FIG. 1. Geometry for a sonic crystal with lattice parameter $a$, cylinder radius $r_{0}$, and representative cell of radius $R$.

where ${ }_{0}$ is the density of the host fluid, $f$ is the filling fraction, and ${ }^{-}$is dynamic tortuosity given by ${ }^{29}$

$$
1 \frac{\bar{F}}{j^{-}}
$$

with denoting the high frequency limit of the tortuosity and the functions $\bar{F}$ and ${ }^{-}$defined as

$$
\begin{aligned}
& \bar{F} \quad \frac{1 j \frac{1}{2}-M}{(1 f)} \\
& -\frac{8}{(1 f)^{2}}
\end{aligned}
$$

From these equations, it can be seen that the e ects of the losses arise from the dynamic viscosity , the static flow resistivity and the characteristic viscous length, which is a viscous parameter defined by Johnson et al. ${ }^{29}$. For the density of a porous medium, the losses arise from viscous e ects, and result in an e ective density which contains both a real and imaginary part.

For a lattice of rigid parallel cylinders embedded in an ideal gas, like that illustrated in Fig. 1, the bulk modulus can be written as

$$
\text { e } \quad \frac{P_{0}}{(1 f)} \frac{1}{C_{\text {fiber }}}
$$

where is the ratio of specific heats, $P_{0}$ is the ambient static pressure, and $f$ is the filling fraction. The sound speed can be determined from Eqs. (1) and (6) by $c_{\mathrm{e}} \overline{\mathrm{e}} \mathrm{e}$. In Eq. (6), $C_{\text {fiber }}$ is the dynamic compressibility, which can be obtained from the thermal boundary conditions.

For thermally conducting fibers in air, the conduction through typical solid materials is several orders of magnitude larger than that of air, giving nearly isothermal conditions. Similarly, when the cylinder spacing is large compared with the thermal boundary layer (corresponding to either relatively high frequencies or low filling fractions), any thermal interaction with the surrounding cylinders can be neglected, which yields adiabatic conditions at the outer radius $R$. Applying 
these thermal boundary conditions, the dynamic compressibility can be obtained, ${ }^{30}$

$$
\begin{aligned}
& C_{\text {fiber }} \quad 1 \quad(\quad 1) \frac{2 f}{(1 f)} \bar{H}
\end{aligned}
$$

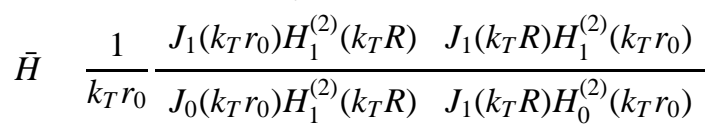

where $H_{m}^{(2)}$ is the $m^{\text {th }}$ order Hankel function of the second kind, $r_{0}$ is the cylinder radius, $R$ is the radius defined by the filling fraction $f \quad\left(r_{0} R\right)^{2}$, and the thermal wavenumber $k_{\mathrm{T}} \quad(1 j) \overline{\mathrm{Pr}}$. The thermal e ects are quantified by the the Prandtl number $\operatorname{Pr} \quad C_{p}$ and the viscous boundary layer is defined as $\overline{(2)\left({ }_{0}\right)}$, where is the viscosity, is the thermal conductivity and $C_{p}$ is the specific heat capacity. It can be seen that the ects of the losses arise from both viscous and thermal e ects, leading to a complex value for the bulk modulus.

\section{Formulation of relevant model parameters}

Due to the random nature of the fibrous media which has traditionally been examined for absorptive acoustic materials, the existing literature has focused on the case of low volume fractions (often on the order of a few percent), using parameters which often require experimental characterization of specific samples since the precise microstructure is not known ${ }^{30-34}$. For such naturally occurring materials, higher volume fractions without a precisely arranged microstructure will tend to clump and intersect, creating what would essentially appear like pores. As a result, the situations of moderate to high concentrations of fibrous porous media have typically been neglected. For lossy sonic crystals, however, these closely packed arrangements are of particular interest, and represent the exact microstructure that one wishes to examine. The three relevant model parameters characterizing the viscous and thermal e ects of the lattice structure are: the viscous characteristic length , the tortuosity and the flow resistivity.

The viscous characteristic length is a metric of the viscous e ects proposed by Johnson et al. ${ }^{29}$. Evaluation of this quantity analytically for the viscous fluid flow around a rigid cylinder yields ${ }^{28}$

$$
\frac{r_{0}}{2 f}\left(1 \quad f^{2}\right)
$$

Note that for small filling fractions, Eq. (9) yields $\quad r_{0}(2 f)$, the same as that obtained by Allard and Champoux ${ }^{28}$. However, for the moderate to high filling fractions that can be achieved using sonic crystals, the higher precision of the exact expression given by Eq. (9) is necessary to accurately describe the acoustic performance.

In the context of sonic crystals, the unique homogenized bulk properties arise from dynamic e ects, and thus for the static flow resistivity it is more appropriate to consider this as a quasi-static condition of low but non-zero oscillatory flow.
For a lattice of parallel rigid cylinders, an expression for the flow resistivity of a structured lattice has been derived by Tarnow $^{35}$

$$
\begin{array}{lllll}
\multicolumn{4}{c}{4 f} \\
\hline r_{0}^{2} & \frac{1}{2} \ln f & \frac{3}{4} & f & \frac{1}{4} f^{2}
\end{array}
$$

which is equivalent to the earlier solution derived for a square lattice following a similar approach by Kuwabara ${ }^{36}$. In both cases, the solution was developed by using a circular representative volume of fluid surrounding each cylinder (illustrated in Fig. 1), and assuming free conditions at the boundary of each cell.

For parallel cylindrical lattices, Tournat et al. ${ }^{37}$ derived an expression for the tortuosity, $1 f$. Although originally developed as an approximate solution valid only for small filling fractions, this solution holds for all filling fractions in the absence of multiple scattering e ects. This can be seen by comparing Eq. (1) in the limit of zero viscosity to the lossless quasi-static dynamic density for a sonic crystal, given by ${ }^{6,38}$

$$
\text { e } 0 \quad 0 \frac{1 \quad f}{1 f f}
$$

\section{Effective density of a 2D sonic crystal with losses}

With the expressions for , and presented above, the complex e ective density given by Eq. (1) for a sonic crystal with viscous losses can be written as

$$
\begin{aligned}
& \text { e } \quad \frac{\frac{1 f}{1 f} 1 j \frac{\bar{F}_{\mathrm{sc}}}{\bar{F}_{\mathrm{sc}}}}{1 j \frac{1}{2}{ }_{\mathrm{sc}} M_{\mathrm{sc}}} \\
& { }_{\text {sc }} \frac{1}{2 f\left(\frac{-}{r_{0}}\right)^{2}} \frac{1 f}{1 f} \quad \frac{1}{2} \ln f \quad \frac{3}{4} \quad f \quad \frac{1}{4} f^{2} \\
& M_{\mathrm{sc}} \quad \frac{8 f}{1 f^{2}} \frac{1 f}{1 f} \quad \frac{1}{2} \ln f \quad \frac{3}{4} \quad f \quad \frac{1}{4} f^{2}
\end{aligned}
$$

From these equations, it is clear that besides the host fluid density, the only parameters that a ect the density are the filling fraction $f$ and the ratio of the viscous boundary layer thickness to the cylinder radius $r_{0}$. From the definition of , it can be seen that this term includes all the relevant viscous e ects and the frequency dependence. Unlike unstructured porous media, which require estimated or experimentally determined scaling parameters ${ }^{39}$, there are no free parameters required for modeling the bulk e ective properties of a lossy sonic crystal. Therefore, the expression presented above for the complex density of a sonic crystal is an explicit expression in terms of the host density and filling fraction, with all the viscous and dispersive e ects accounted for by a single dimensionless parameter, $r_{0}$, which can be calculated based on the frequency and the properties of the viscous host fluid. 


\section{Effective bulk modulus of a 2D sonic crystal with losses}

In a similar manner, Eqs. (6)-(8) can be used to express the bulk modulus of a sonic crystal with viscous losses,

$$
\begin{array}{rl}
\text { e } & \frac{P_{0}}{(1 f)} \frac{1}{C_{\mathrm{sc}}} \\
C_{\mathrm{sc}} & 1 \quad(\quad 1) \frac{2 f}{(1 f)} \bar{H}
\end{array}
$$

As with the complex density, the complex bulk modulus is a function only of the properties of the host fluid (including thermal properties), the filling fraction and the parameter $r_{0}$.

\section{B. Parametric representation of lossy sonic crystals}

From Eqs. (8), (12)-(17), theoretical values for the complex density and bulk modulus can be obtained. These expressions do not contain any empirically derived coe cients, and for a given host fluid can completely describe any combination of lattice geometries and frequency using two independent parameters: the filling fraction $f$ and the normalized viscous boundary layer thickness, $r_{0}$. Therefore, it is possible to create parametric plots of the e ective sonic crystal properties, which can encompass the entire range of possible e ective properties for 2D sonic crystals with thermoviscous losses, for a given host fluid. Use of such plots allow for the design and interpretation of sonic crystal e ective properties when thermoviscous losses are present, and enable one to better characterize the potential absorption properties of a sonic crystal.

Parametric plots are illustrated in Fig. 2 for the complex density, sound speed and bulk modulus of a 2D sonic crystal in air, as a function of the filling fraction and $r_{0}$. The parameter space has been limited to values where the thermal and viscous boundary layers are su ciently small so as they do not touch the boundary layer of the adjacent cylinders. The limiting case where the boundary layers touch is denoted by a solid black line. FIG. 2(a), (c) and (e) shows the real part of the property and Fig. 2(b), (d) and (f) shows the loss factor (imaginary part divided by the real part) on a color scale, ranging from low values (dark) to high values (light). Note that while fibrous porous materials have been extensively utilized for sound absorbing applications, these have traditionally been limited to low filling fractions, on the order of a few percent, which represents the left-most region of the plots.

Expanding the parameter space to include the higher filling fractions made possible by the structured lattice of the sonic crystals, one can identify several desirable features which could be utilized for acoustic absorbers. In particular, it is observed that there is a broad region across the moderate to high filling fractions where the loss factor is large, and in the case of the density approaches unity, compared with very small values for the region covered by traditional fibrous porous absorbers. In addition, from Fig. 2(b) it can be seen that significant reductions in the real part of the sound speed, which represents the speed of the wave through the homogenized sonic
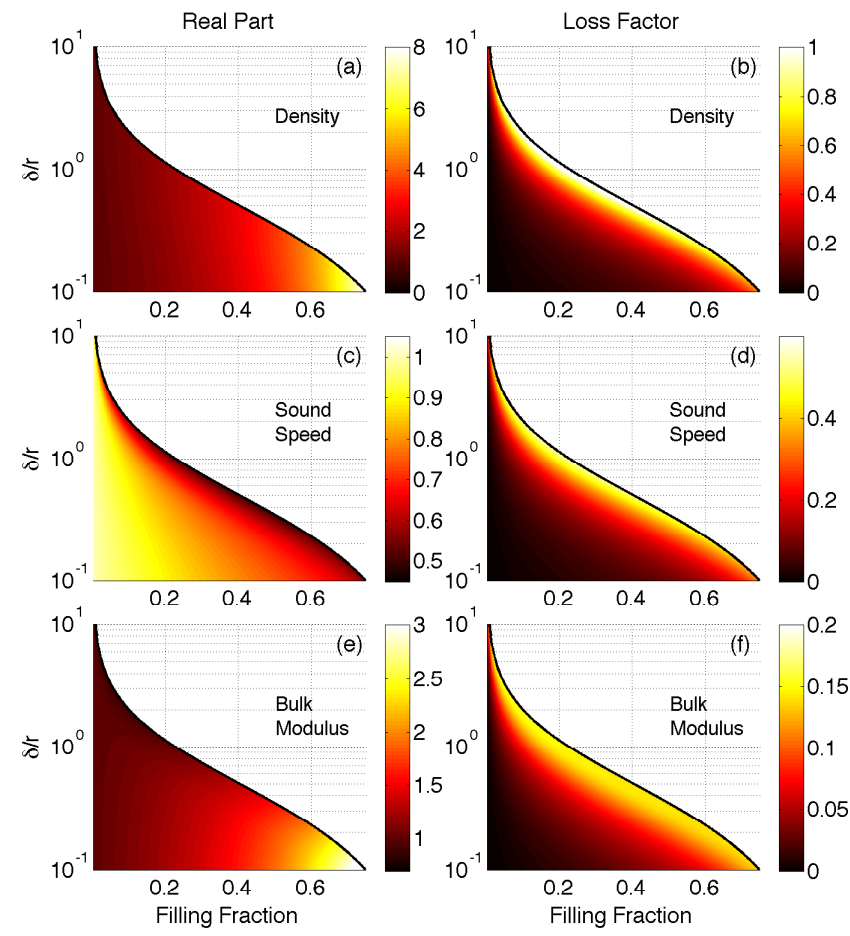

FIG. 2. (Color online) Intensity plots of the real part and loss factor for the complex e ective density, sound speed and bulk modulus. The color scale of the plots ranges from low values (dark) to high values (light). All the values are normalized to the corresponding ones of the air background.

\begin{tabular}{ccccc}
\hline \hline Sample & $r_{0}(\mathrm{~mm})$ & $a(\mathrm{~mm})$ & Length, $L(\mathrm{~mm})$ & Filling fraction, $f$ \\
\hline $\mathrm{A}$ & 10 & 25 & 425 & 0541 \\
$\mathrm{~B}$ & 10 & 38 & 431 & 0234 \\
$\mathrm{C}$ & 10 & 50 & 450 & 0134 \\
\hline \hline
\end{tabular}

TABLE I. Lattice properties for the three sonic crystal samples fabricated in this work.

crystal, occur at moderate to high filling fractions. Although this does not change the absorption per cycle, it does a ect the wavelength of the sound passing through the absorber. Decreasing the sound speed, as shown in Fig. 2(b), will decrease the wavelength, and therefore lead to an absorber which appears acoustically "thicker" and thereby increasing the total absorption.

\section{Comparison of results with Comsol}

To verify the theoretical formulation developed in Section II A, the complex density and bulk modulus are compared with Comsol simulations. In the Comsol models, the cylinders are assumed to be rigid, and a thermoviscous host fluid with the properties of air is used. Although the dimensionless parameter $r_{0}$ is utilized for the theoretical analysis, the use 

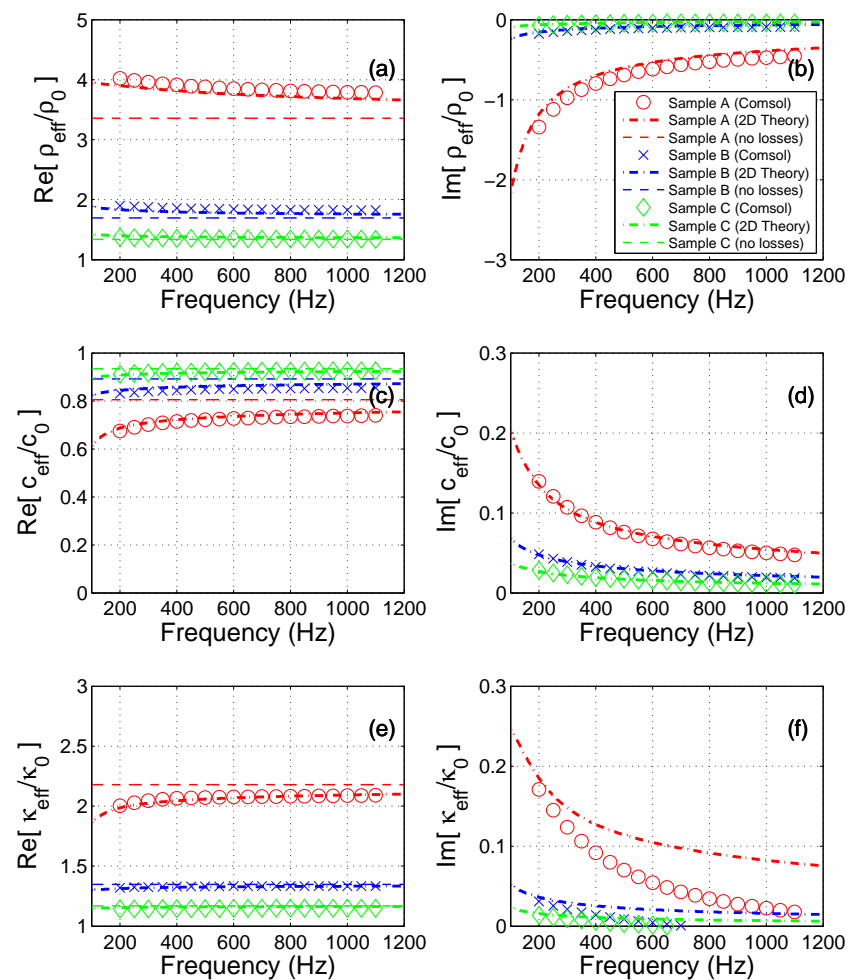

FIG. 3. (Color online) Comparison of theoretical results and Comsol for the real and imaginary parts of the density and bulk modulus of a sonic crystal with $r_{0} 1 \mathrm{~mm}$, for Sample A, B, and C given in Table I, shown in red, blue and green, respectively. Theoretical results for the 2D sonic crystal configurations are obtained from Eqs. (12) and (16).

of Comsol requires specific dimensions and a corresponding frequency range for the acoustical modeling to be performed. Based on the definition of,$\quad r_{0}$ can be calculated for a specific fluid (in this case air) and frequency range and the cylinder radius $r_{0}$.

Results calculated from Eqs. (12) and (16) are compared with Comsol simulations in Fig. 3, for Samples A, B, and C, the dimensions of which are listed in Table I. For reference, e ective properties for the lossless case are shown in Fig. 3(a), (c) and (e) and denoted by a dashed line. In this figure, it is clear that there is excellent agreement between the theoretical model developed here and the Comsol data, for both the real and imaginary parts of the density, sound speed and bulk modulus. Conversely, the e ective properties for the lossless cases fail to capture the trends in the data for even the real part of the e ective properties as a function of frequency, and the overall magnitude deviates from that of either the theory with losses or Comsol at higher filling fractions. Based on these results, the theoretical formulation with losses provides a relatively simple yet accurate explicit formulation for the e ective properties of a lossy sonic crystal structure.

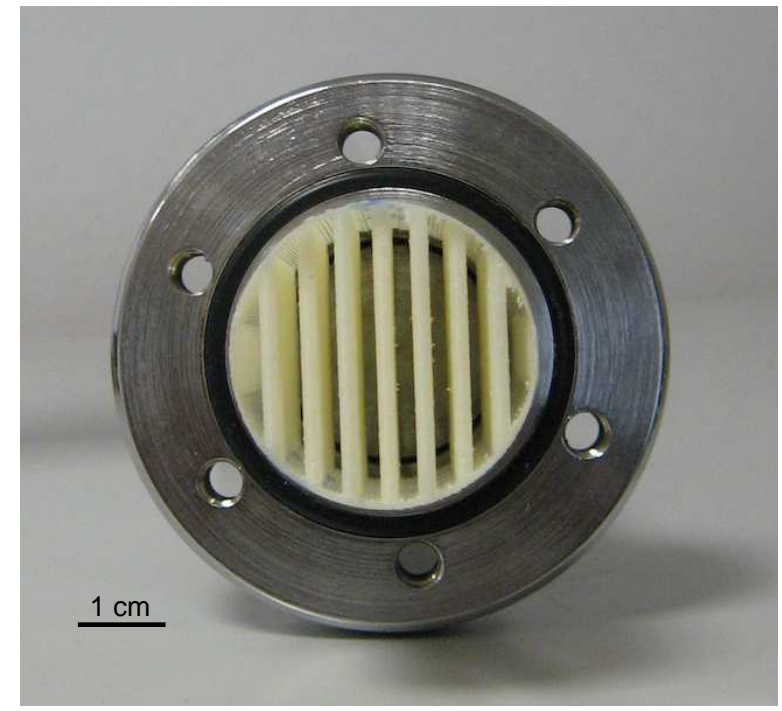

FIG. 4. (Color online) Photograph of a confined sonic crystal sample inside an acoustic impedance tube. The plastic sheath surrounding the sonic crystal allows its insertion in the tube.

\section{MODIFIED FORMULATION FOR CONFINED SONIC CRYSTALS}

Through the process of producing a finite sized sonic crystal sample which can be investigated and characterized, a confined sonic crystal or acoustic metamaterial will inevitably be created due to the walls and structure enclosing it. Although creating confined sonic crystals can be done as a design choice, in many practical cases this occurs as a result of using standard acoustic testing techniques, such as an impedance tube. An illustration of a confined sonic crystal sample situated inside an impedance tube is shown in Fig. 4. In this section, modifications to the theoretical results presented in Section II will be discussed, which can account for these e ects.

The presence of the walls within the sonic crystal sample, as with the air-filled portion of the impedance tube, will be affected by the viscous and thermal boundary layers emanating from these surfaces. However, these boundary layers within the sample will be a ected by the e ective viscous and thermal properties of the sonic crystal sample, which consists of both solid cylinders and the surrounding air. At moderate to high filling fractions, these e ective thermoviscous properties can vary significantly from those of ambient air, leading to observable di erences in the e ective sonic crystal properties. In Section III A, expressions for e ective thermal and viscous properties are presented, based on e ective medium theory. In Section III B, these e ective medium properties are used to determine the appropriate thermal boundary conditions and a revised expression for the dynamic compressibility and bulk modulus for a confined sonic crystal is developed. 


\begin{tabular}{lcc}
\hline \hline Material properties & Air & ABS plastic \\
\hline Density, & $121 \mathrm{~kg} \mathrm{~m}^{3}$ & $1050 \mathrm{~kg} \mathrm{~m}^{3}$ \\
Bulk modulus, & $142 \mathrm{kPa}$ & $24 \mathrm{GPa}$ \\
Shear modulus, & - & $081 \mathrm{GPa}$ \\
Compressional wave speed, $c$ & $343 \mathrm{~m} \mathrm{~s}$ & $1834 \mathrm{~m} \mathrm{~s}$ \\
Thermal conductivity, & $00263 \mathrm{~W} \mathrm{~m} \mathrm{~K}$ & $017 \mathrm{~W} \mathrm{~m} \mathrm{~K}$ \\
Specific heat capacity, $\mathrm{C}_{\mathrm{p}}$ & $1000 \mathrm{~J} \mathrm{~kg} \mathrm{~K}$ & $1300 \mathrm{~J} \mathrm{~kg} \mathrm{~K}$ \\
Ratio of specific heats, & 14 & 10 \\
Viscosity, & $185 \mathrm{~Pa} \mathrm{~s}$ & - \\
\hline \hline
\end{tabular}

TABLE II. Physical properties of air and ABS plastic used for the fabricated sonic crystal samples examined in Section V.

\section{A. Effective thermal and viscous properties}

For moderate to high concentrations of inclusions, the bulk properties of the e ective medium can be significantly influenced by the number and proximity of the inclusions to one another. While this has not traditionally been a factor for previous works on unstructured fibrous porous media in air, this e ect has been studied quite extensively for elastic composite structures and for fluid emulsions.

To determine the necessary e ective properties, recall that the Prandtl number Pr was used to quantify the relative strength of the thermal and viscous e ects, which is determined by the specific heat capacity, thermal conductivity and viscosity of the medium. The e ective value for the heat capacity, which is given by the product $\mathrm{C}_{\mathrm{p}}$, is simply ${ }^{40}$

$$
\mathrm{C}_{\mathrm{p}_{\mathrm{e}}} \quad f \quad \mathrm{C}_{\mathrm{p}_{\text {inc }}}\left(\begin{array}{llll}
1 & f
\end{array}\right) \mathrm{C}_{\mathrm{p}_{\text {fluid }}}
$$

where the subscripts "inc" and "fluid" refer to the inclusion and fluid components, respectively. Material properties for air and the ABS plastic used for the fabricated samples examined in Section V are given in Table II. Note that the density of the cylinder is several orders of magnitude larger than that of air, while the specific heat capacity for most solids are of the same order of magnitude to those for air, so the e ective specific

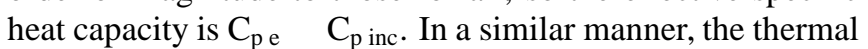
conductivity along the axial direction of the ective medium (the vertical direction as shown in Fig. 4) can be determined using the rule of mixtures, which yields ${ }^{41}$

$$
\text { e } \quad f \text { inc }(1 \quad f) \text { fluid }
$$

In addition to the thermal properties, the e ective viscosity will be a ected by the presence of the inclusions. This wellknown phenomenon has been traditionally examined for suspensions, and the classic solution for a low concentration of rigid spheres in a viscous fluid is attributed to Einstein ${ }^{42}$. Although this expression is extensively used, its applicability is limited to objects with a spherical shape. For the case of rigid cylinders in a viscous fluid, the e ective viscosity can be determined by analogy with elastic composites, which yields ${ }^{41}$

$$
\left(\begin{array}{ll}
1 & 2 f
\end{array}\right)
$$

The e ective density for a confined sonic crystal is given by the same expression as for the unconfined case described by Eq. (12), except with an increase in the e ective viscosity, as described by Eq. (20). Note that this increased viscosity corresponds to higher flow resistivity according to Eq. (10), and therefore an increase in the imaginary part of the density and ultimately higher losses.

\section{B. Effective bulk modulus for confined sonic crystals}

For a confined sonic crystal, the presence of the surrounding surfaces will a ect how the thermoviscous boundary layers interact with the cylinders and the resulting e ective bulk modulus of the homogenized structure. Although the fundamental equations are the same as for the unconfined sonic crystal, the cylinders within the confined sonic crystal will experience a di erent thermal boundary condition at $r \quad R$, the outer radius of the unit cell. For high filling fraction applications relative to the boundary layer thickness, the outer unit cell boundary conditions are often set equal to those of the e ective medium, to compensate for the net interaction from the surrounding cylinders ${ }^{40}$. This approach of treating a unit cell surrounded by an e ective homogenized medium is also utilized extensively in e ective medium theory for elastic solids $^{41}$.

In the idealized 2D expressions developed in Section II, it was appropriate to assume that the interactions between cylinders could be neglected, since the thermal and viscous e ects were confined to the relatively thin boundary layers close to each cylinder. For the confined sonic crystal, however, viscous and thermal boundary layers emanate from the walls of the confining structure, with thermoviscous properties of the effective medium. Even at low filling fractions, these boundary layers can be significantly larger than those in air for thermally conductive cylinders, and under these circumstances it is appropriate to apply thermal boundary conditions at $r \quad R$ equal to those of an e ective homogenized sonic crystal medium. For an e ective homogenized medium surrounding the unit cell containing low to moderate filling fractions of thermally conducting cylinders, this will yield an approximately isothermal boundary condition, for which the expression for the dynamic compressibility becomes

$$
C_{\text {SC conf }} 1 \quad(\quad 1) \frac{2 f}{(1 f)} \bar{H}_{\text {conf }}
$$

where

$$
\begin{aligned}
& \bar{H}_{\text {conf }} \quad k_{T} r_{0} J_{1}\left(k_{T} r_{0}\right) \quad k_{T} R J_{1}\left(k_{T} R\right) \quad H_{0}^{(2)}\left(k_{T} r_{0}\right) \quad H_{0}^{(2)}\left(k_{T} R\right) \\
& \begin{array}{llll}
J_{0}\left(k_{T} r_{0}\right) & J_{0}\left(k_{T} R\right) & k_{T} r_{0} H_{1}^{(2)}\left(k_{T} r_{0}\right) & k_{T} R H_{1}^{(2)}\left(k_{T} R\right)
\end{array} \\
& J_{0}\left(k_{T} r_{0}\right) H_{0}^{(2)}\left(k_{T} r_{0}\right) \quad H_{0}^{(2)}\left(k_{T} R\right) \\
& J_{0}\left(k_{T} r_{0}\right) \quad J_{0}\left(k_{T} R\right) H_{0}^{(2)}\left(k_{T} r_{0}\right)
\end{aligned}
$$

Therefore, the bulk modulus of a confined sonic crystal can be described by Eq. (16), with the dynamic compressibility 
given by Eqs. (21) and (22). Although Eq. (22) is a somewhat complicated expression, it is expected that a decrease in the e ective bulk modulus will be observed due to the change from adiabatic to isothermal conditions.

\section{ACOUSTIC METAMATERIAL USING ALTERNATING LOSSY SONIC CRYSTAL LAYERS}

Recent work has examined anisotropic acoustic metamaterials theoretically, numerically and experimentally ${ }^{14,15}$. Despite these thorough investigations and demonstrations of realizable structures, such works have neglected thermoviscous losses due to a primary focus on broadband, nonresonant acoustic metamaterials which operate without any appreciable losses. Comparison of theoretical results with Comsol simulations for 2D sonic crystals (presented in Fig. 3) shows that significant di erences were observed between e ective properties obtained assuming a lossless host fluid and those which include thermoviscous losses. These di erences resulted in a non-zero (and at some frequencies quite large) imaginary part, and also resulted in incorrect trends predicted by the lossless theory, including underestimating the real part of the density and overestimating the real part of the bulk modulus. Therefore, in the following sections an analysis of an anisotropic acoustic metamaterial will be performed. The theoretical framework for this will be discussed in Section IV A, in which the anisotropic acoustic metamaterial will be treated as a system of alternating e ective fluid layers, with the properties of each e ective fluid layer simply being the complex e ective properties determined from the homogenization process of a uniform sonic crystal. In Section IV B, the theoretical formulation is compared with 2D Comsol simulations with thermoviscous losses for a realizable configuration.

\section{A. Theoretical formulation}

In this section, the formulation for the e ective properties of an acoustic metamaterial with complex anisotropic inertia will be examined, which consists of an alternating-layer arrangement of sonic crystal lattices. The anisotropy in the inertia arises from di erences in the e ective density of the homogenized structure at di erent orientations of the structure. For the impedance tube testing under investigation in this work, of particular interest is the analysis relating to normal incidence plane waves for two specific configurations, where the impinging wave is either parallel or perpendicular to the sonic crystal layers, which are illustrated in Fig. 5(a) and (b), respectively.

When the acoustic metamaterial is oriented perpendicular to the incident wave, the e ective density and bulk modulus of an alternating layer structure is given by the harmonic average of the quantities, namely, ${ }^{43}$

$$
\text { e } \quad \frac{d_{1}}{d_{\text {tot }}} \frac{1}{1} \quad \frac{d_{2}}{d_{\text {tot }}} \frac{1}{2}
$$

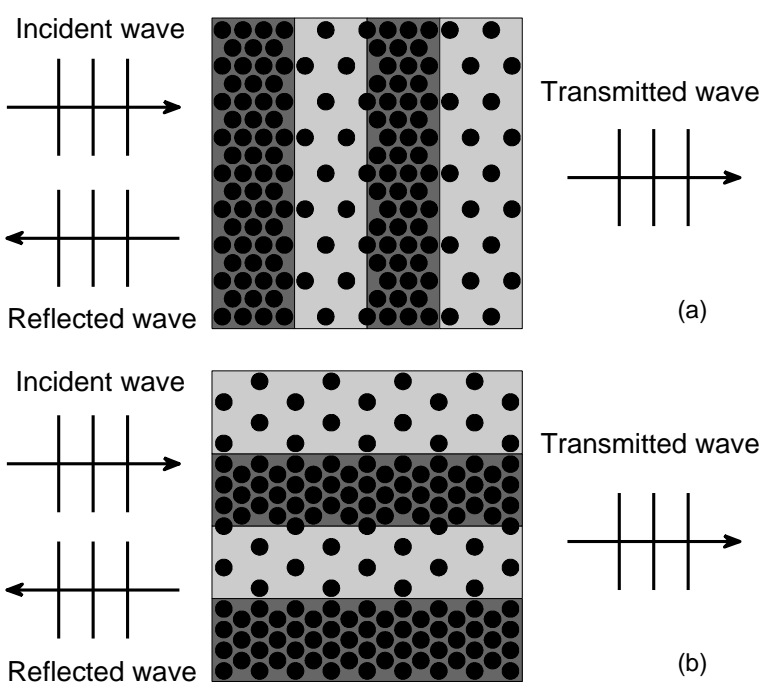

FIG. 5. (a) Parallel and (b) perpendicular configurations of the anisotropic acoustic metamaterial examined in this work, which consists of two sets of alternating sonic crystal layers. The two sonic crystal layers behave as e ective fluids, which have the homogenized properties of Samples A and C given in Table I, and are denoted by dark and light gray, respectively.

$$
\text { e } \quad \frac{d_{1}}{d_{\mathrm{tot}}} \frac{1}{1} \quad \frac{d_{2}}{d_{\mathrm{tot}}} \frac{1}{2}
$$

where $d_{\text {tot }} \quad d_{1} \quad d_{2}$, and the subscripts 1 and 2 refer to the first and second alternating fluids layers. To determine the e ective properties of the acoustic metamaterial, a two-step homogenization process will be performed. First, each sonic crystal lattice will be homogenized to create an e ective fluid layer, using the methods described in Section II for a 2D sonic crystal, or for the results developed for a confined sonic crystal discussed in Section III. Second, these e ective fluid layers will be homogenized to obtain the e ective properties of the acoustic metamaterial in both the parallel and perpendicular orientations of the sonic crystal layers.

For a multilayered arrangement of an arbitrary number of fluid layers, this analysis can be performed using the impedance and pressure translation theorems to obtain the input specific acoustic impedance, $Z_{\text {in }}$, and normalized acoustic pressure, $P$, which are given by 44,45

$$
\begin{aligned}
& Z_{\text {in }}\left(x_{i}\right) \quad Z_{i} \frac{Z_{\text {in }}\left(x_{i 1}\right) \cos k_{i} d_{i} \quad j Z_{i} \sin k_{i} d_{i}}{Z_{i} \cos k_{i} d_{i} \quad j Z_{\text {in }}\left(x_{i 1}\right) \sin k_{i} d_{i}} \\
& P\left(x_{i 1}\right) \quad P\left(x_{i}\right) \cos k_{i} d_{i} \quad j \frac{Z_{i}}{Z_{\text {in }}\left(x_{i 1}\right)} \sin k_{i} d_{i}
\end{aligned}
$$

where $x_{i}$ is the position of the $i^{\text {th }}$ fluid interface, $d_{i} \quad x_{i} x_{i}$ is the thickness of the $i^{\text {th }}$ layer, $k_{i}$ is the wavenumber of the $i^{\text {th }}$ layer, and $Z_{i}$ is the specific acoustic impedance of the $i^{\text {th }}$ layer. Implementation of Eqs. (25) and (26) can be achieved by solving for the input impedance first, and then evaluating the acoustic pressure. Starting from the last layer (which radiates 
into air) and working backwards yields the input impedance at each successive layer, until the input impedance at the first layer, $Z_{\text {in }}(0)$, is determined. Likewise, the normalized acoustic pressure can then be determined, starting at the first layer and working forward, until the pressure at the last layer is determined, denoted by $P(L)$, where $L$ is the total length of the multilayer structure. From these two values, the pressure reflection coe cient, , and transmission coe cient, , can be determined,

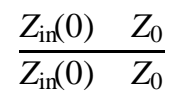

The e ective homogenized properties of the ensemble structure can be determined using and for a single e ective fluid layer with specific acoustic impedance $Z_{\mathrm{e}}$, wavenumber $k_{\mathrm{e}}$ and length $L$. Using well-known physical acoustic solutions for a single fluid layer ${ }^{46}$, one can obtain expressions for the ective properties $Z_{\mathrm{e}}$ and $k_{\mathrm{e}}$, such that

$$
\begin{aligned}
& Z_{\mathrm{e}} \frac{1 \quad \cos k_{\mathrm{e}} L}{j \sin k_{\mathrm{e}} L} \\
& k_{\mathrm{e}} \quad \frac{1}{L} \cos ^{1} \frac{1 \quad 2 \quad 2}{2}
\end{aligned}
$$

where $\cos { }^{1}$ denotes the inverse of the cosine function. A similar result has been previously derived Fokin et al. ${ }^{47}$, though in this previous work some uncertainty arises due to the periodic but nonunique solution that results from evaluating the $\cos 1$ function in Eq. (29). Alternatively, Eq. (29) can be evaluated by unwrapping the solution for the $\cos ^{1}$ function, such as by using the method proposed by Baccigalupi ${ }^{48}$.

The e ective density, sound speed, and bulk modulus can be determined from Eqs. (28) and (29), such that

$$
\text { e } \quad Z_{\mathrm{e}} \frac{k_{\mathrm{e}}}{k_{\mathrm{e}}} \quad c_{\mathrm{e}} \quad \text { e } \quad Z_{\mathrm{e}} \overline{k_{\mathrm{e}}}
$$

In the low frequency limit, the expressions for the e ective density and bulk modulus for alternating fluid layers oriented in the parallel direction reduces to

$$
\begin{array}{ll}
\text { e } \frac{d_{1}}{d_{\text {tot }}} 1 \frac{d_{2}}{d_{\text {tot }}} 2 \\
\text { e } \frac{d_{1}}{d_{\text {tot }}} \frac{1}{1} \frac{d_{2}}{d_{\text {tot }}} \frac{1}{2}
\end{array}
$$

which corresponds to the previously established results by Schoenberg and Sen ${ }^{43}$ extensively used in anisotropic metamaterial analysis ${ }^{7}$. Note that in this case the e ective bulk modulus reduces to the same value as in the perpendicular orientation given by Eq. (24), so that the anisotropy occurs only in the density in the quasi-static limit.

\section{B. Comparison of results with Comsol}

To further examine the theoretical formulation presented in Section IV A, Comsol was used to determine the e ective properties of an acoustic metamaterial with complex
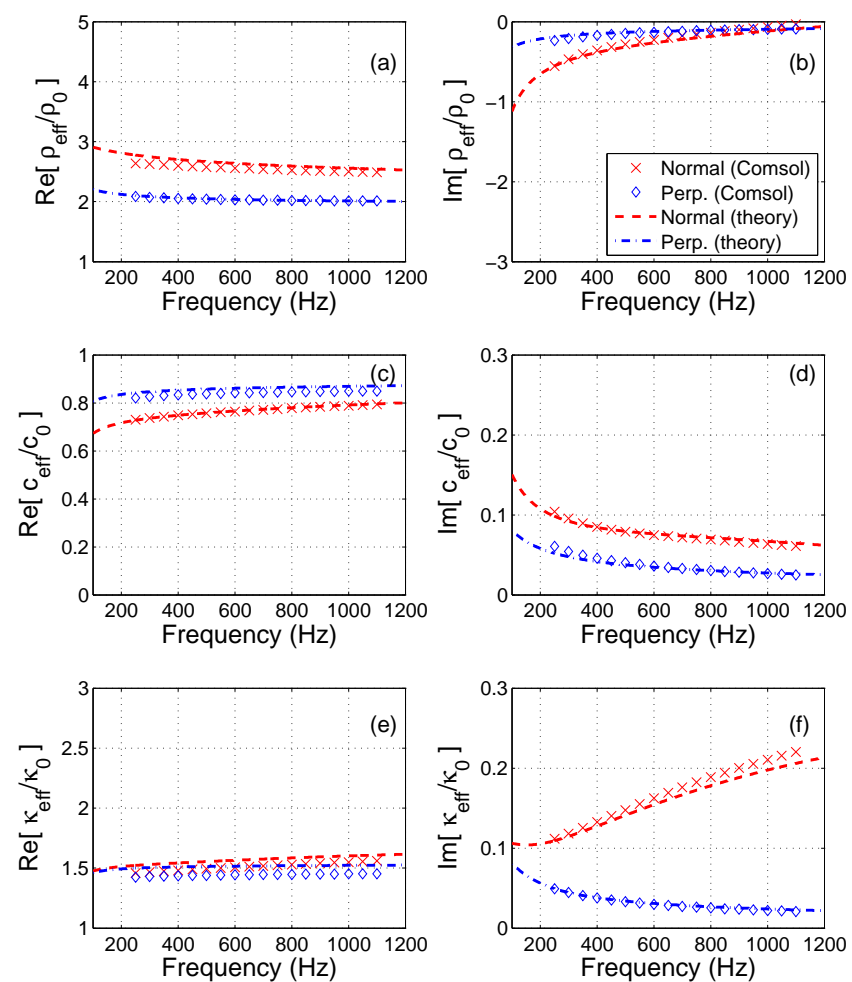

FIG. 6. (Color online) Comparison of theoretical results and Comsol for the real and imaginary parts of the density and bulk modulus of a sonic crystal with $r_{0} 1 \mathrm{~mm}$, for the anisotropic acoustic metamaterial configuration illustrated in FIG. 5.

anisotropic inertia, for the configuration shown in Fig. 5. The results obtained using Comsol are presented in Fig. 6 for the incident wave parallel (x's) and perpendicular (circles) to the sonic crystal layers, consisting of rigid cylinders in air with thermoviscous losses. Theoretical values for the parallel and perpendicular configurations represented by the dashed and dash-dotted lines, respectively, are in excellent agreement with those obtained with Comsol, for both the real and imaginary part of the e ective properties.

In Fig. 6(a) and (b), the anisotropy in the density is clearly seen, with the results for the parallel direction noticeably higher than that for the perpendicular case. This anisotropy in the complex density is also apparent in the results for the sound speed illustrated in Fig. 6(c) and (d), which shows a similar trend. In Fig. 6(e), there is only a slight di erence in the real part of the e ective bulk modulus between the two configurations, as expected by the quasi-static results given by Eqs. (24) and (32). Interestingly, a more noticeable di erence between the parallel and perpendicular configurations is observed in the imaginary part of the bulk modulus shown in Fig. 6(f), a trend that is captured using the theory by retaining the full expressions presented in Section IV A, rather than the quasi-static approximations. From this observation, it is clear that to correctly account for the losses, it is important to retain the slightly more complicated general expression given by Eqs. (28)-(30).

From a theoretical point of view, an anisotropic acoustic 
absorber can be obtained from any alternating arrangement of lossy fluids. However, realization of such an arrangement using actual fluids is impractical, particularly for the airborne sound absorbers examined in this work, and thus requires the use of e ective lossy fluid structures. Furthermore, the range of e ective fluid properties using traditional randomly oriented fibrous media is quite limited, as discussed in Section II B. Alternatively, the use of high filling fraction sonic crystals enable a significant increase in the range of the effective density and bulk modulus, in addition to the increased loss factors observed in in Section IIB. This allows for the realization of large inertial anisotropy like that theoretically and numerically illustrated in Fig. 6.

\section{EXPERIMENTAL RESULTS}

To verify the theoretical model for confined sonic crystals, several samples were fabricated and experimentally tested using a standard circular cross-section acoustic impedance tube. The inner diameter of the impedance tube is $3.5 \mathrm{~cm}$, and the end of the tube is terminated with fiber glass insulation to provide an anechoic termination. Noise is generated and transmitted using a electromechanical driver, and measured using 0.50 inch $(1.27 \mathrm{~cm})$ diameter G.R.A.S. condenser microphones. The microphones are arranged in a standard 4microphone configuration ${ }^{49}$, allowing for the magnitude and phase of both the reflection and transmission pressure coe cients to be directly determined using a transfer-matrix method $^{50}$, from which the complex impedance and wavenumber were obtained for the range $300-2000 \mathrm{~Hz}$ as done in previous experimental work on sonic crystal e ective properties ${ }^{51}$.

Some practical obstacles which arise from the fabrication of samples are examined and accounted for in the model, including the e ects of a thin plastic sheath around the sonic crystal used for structural support in Section V A. Experimental results for uniform sonic crystal lattices (which demonstrate isotropic e ective inertia) for the three filling fractions described in Table I are presented in Section V B. The multilayer arrangement of these uniform sonic crystal lattices, which enables anisotropic inertia, are used to create the acoustic metamaterial absorber and is presented in Section $\mathrm{V} \mathrm{C}$.

\section{A. Effects of sample sheath}

In this section the e ects of the plastic sheath used to provide structural support for the fabrication and experimental testing of the samples will be discussed. Although the plastic sheath is quite thin compared with the radius of the impedance tube (as seen in Fig. 4), the presence of this sheath leads to two main e ects: an increase in the measured e ective acoustic impedance, and it leads to a layer of air which increases the e ective density and decreases the e ective bulk modulus the sample.

The addition of the plastic sheath to the sonic crystal will lead to an increase in the measurable properties of the sonic crystal sample. Assuming that the sheath appears rigid relative to the surrounding air, this will lead to increase in $\mathrm{e}$ and e , given by

$$
\begin{array}{lllll}
\text { e sheath } & \text { e } & 1 & \text { sheath } & 1 \\
\text { e sheath } & \text { e } & 1 & \text { sheath } & 1
\end{array}
$$

where sheath $l_{\text {sheath }} R_{\text {tube }}$, with $l_{\text {sheath }}$ and $R_{\text {tube }}$ denoting the sheath thickness and the radius of the tube, respectively. In addition to the sheath encasing the sonic crystal, a slight lip (where the sheath extended slightly past the cylinder) was present due to the fabrication process. The result of this lip is a thin layer of air adjacent to the front and back of sonic crystal sample. The observed e ective properties can be quantified by assuming that the resulting thin air layer acts like an acoustic lumped element, in which case

$$
\begin{array}{llllll}
\text { e } & \text { lip } & \text { e } & 0 & \text { lip } \\
\text { e } & \text { lip } & \frac{1}{\mathrm{e}} & & \frac{\text { lip }}{0} &
\end{array}
$$

where lip $l_{\text {lip }} R_{\text {tube. }}$ The nominal sheath thickness, $l_{\text {sheath }}$, and sheath lip, $l_{\text {lip }}$, in each case tested in this work was 05 $\mathrm{mm}$ and $1 \mathrm{~mm}$, respectively.

From Eqs. (33)-(36) it can be observed that the sheath and the sheath lip will increase the measured e ective density. For the bulk modulus, the sheath itself will lead to an increase due to the increase in the specific acoustic impedance, though the presence of the sheath lip acts to reduce the e ective bulk modulus. Although which factor dominates depends on the precise thicknesses of the sheath and sheath lip, it is clear from Eq. (36) that the observable e ective bulk modulus resulting from the sheath lip will depend on the relative magnitude of the e ective bulk modulus of the sonic crystal compared with that of the ambient air. Thus, the presence of the sheath lip will be amplified as the filling fraction increase, and therefore one would expect this e ect to dominate at higher filling fractions, where e 0 .

\section{B. Experimental results with isotropic inertia}

The sonic crystal samples were created using a commercial 3D printer out of ABS plastic for several di erent configurations, which are listed in Table I and cover a wide range of filling fractions. The three sonic crystal samples described in Table I, consisting of a single uniform arrangement with constant lattice parameter, were constructed to verify the results of the modeling of the confined sonic crystals, based on the formulations presented in Section III. A photograph of a sample mounted in the impedance tube is shown in Fig. 4. In this figure, the thin plastic sheath surrounding the sonic crystal of the test sample can be seen, which was necessary for structural support to ensure the cylinders remained properly aligned.

Figure 7 shows the experimental results for the complex density, sound speed and bulk modulus for Samples A, B, and $\mathrm{C}$. For comparison, two theoretical models of the sonic crystal samples are presented: the first being the $2 \mathrm{D}$ sonic 

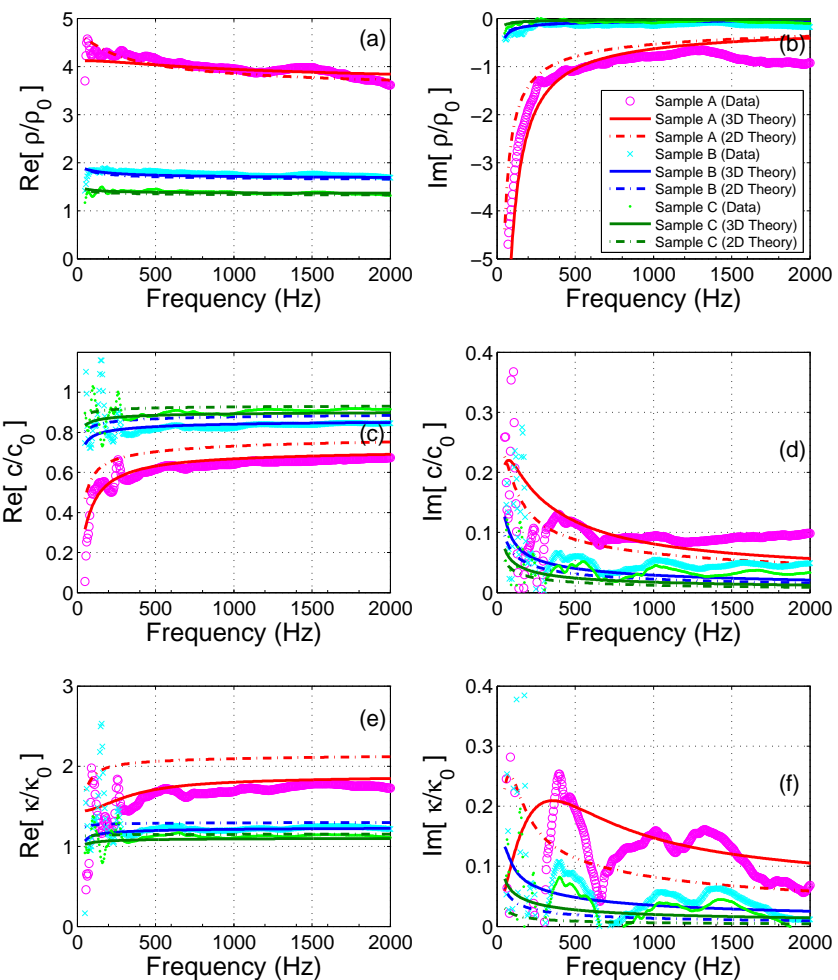

FIG. 7. (Color online) Comparison of theoretical results and experimental impedance tube data as a function of frequency for the real and imaginary parts of the e ective density and bulk modulus of a sonic crystal with $r_{0} 1 \mathrm{~mm}$, for Sample A, B, and C given in Table I, shown in red, blue and green, respectively. The e ective sonic crystal properties are normalized relative to the properties of air. Theoretical results for the 2D sonic crystal configurations are obtained from Eqs. (12) and (16), and the 3D e ects of the confined sonic crystals are accounted for using Eqs. (18)-(22).

crystal model (also shown in Fig. 3), and second including the modifications for the confined sonic crystal with the effective thermal properties and isothermal boundary conditions for the dynamic compressibility described by Eqs. (21), (20), and (22). For the real and imaginary parts of the density shown in Fig. 7(a) and (b), there is an excellent agreement between both models and the experimental results, with only a slight deviation observed with the unconfined 2D sonic crystal model at the highest filling fraction (Sample A). For the complex sound speed and bulk modulus shown in Fig. 7(c)(f), there is a much more significant di erence between the modeled results, resulting from the di erent thermal boundary conditions used to derive the expressions for the dynamic compressibility, and thus the bulk modulus. In particular, it is observed that the 2D sonic crystal model, which was in excellent agreement with the 2D results presented in Fig. 3, yields a bulk modulus which has a significantly higher real part, with a correspondingly lower imaginary part, than the experimental data. This trend is also observed in the sound speed data as well. However, the theoretical formulation with the modifications for the confined sonic crystal correctly accounts for this decrease in the bulk modulus and increase in the losses
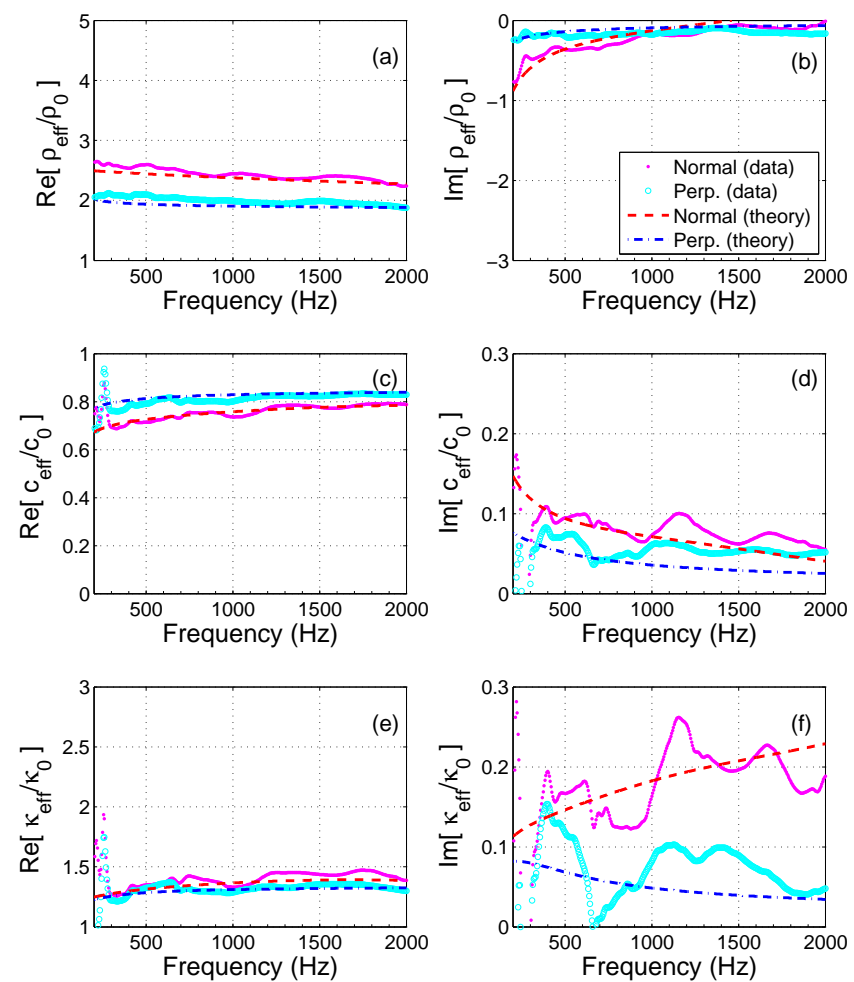

FIG. 8. (Color online) Comparison of theoretical results and experimental impedance tube data for the real and imaginary parts of the density and bulk modulus of a sonic crystal with $r_{0} \quad 1 \mathrm{~mm}$, for the anisotropic acoustic metamaterial configuration illustrated in Fig. 5. The e ective sonic crystal properties are normalized relative to the properties of air. Theoretical results for the acoustic metamaterial are given by Eqs. (23)-(30), with the sonic crystal e ective fluid properties determined from Eqs. (12), (16), and (18)-(22).

(characterized by the imaginary part of the properties), and is in excellent agreement with the experimental results for the entire range of filling fractions examined.

\section{Experimental results with anisotropic inertia}

For the realization of the acoustic metamaterial with complex anisotropic inertia, two samples were constructed using the arrangement illustrated in Fig. 5(a) and (b) to demonstrate the anisotropy in the parallel and perpendicular directions, respectively. As seen in Fig. 5, these samples consist of two sets of alternating sonic crystal layers, containing a high filling fraction layer (dark) and low filling fraction (light). The same experimental setup was used to test these acoustic metamaterial samples, the results for which are presented in Fig. 8. For comparison purpose, theoretical results obtained from Eqs. (28) and (29) are also shown. These results correspond to the ective properties of the structures with parallel (dashed lines) and perpendicular (dash-dotted liens) configurations, which are calculated using the homogenized layer properties based on those for confined sonic crystals (corresponding to the theoretical results presented for Sample A 
and $\mathrm{C}$ in Fig. 7). Upon examination of the data shown in Fig. 8, excellent agreement is observed between the theoretical and experimental results. This corresponds to an accurate description of both the real and imaginary parts of each e ective property, for each orientation. The anisotropic inertia for this acoustic metamaterial is observed in the data through the significantly di erent values in the real and imaginary parts of the e ective density for the parallel and perpendicular orientations. These trends are precisely captured by the theoretical results, for the complex values of the density, sound speed and bulk modulus.

\section{SUMMARY}

This work has reported theoretical and experimental results for the consideration of thermal and viscous losses on the performance of sonic crystals, with filling fractions much larger than traditional porous absorbers. Due to the ordered microstructure, expressions for the complex e ective parameters of sonic crystals can be written with no unknown or empirical coe cients. In addition, it was shown that they can be completely characterized by only the filling fraction and normalized boundary layer thickness. From these results, parametric plots were developed and examined, and highlight desirable characteristics for the enhancement of sound absorption, including loss factors near unity and low bulk moduli. The e ects of a confining structure around a sonic crystal lattice were examined theoretically and experimentally, with the results showing excellent agreement over a wide range of filling fractions and frequencies. A formulation for acoustic metamaterials with complex-valued e ective material properties has been presented, making use of these confined sonic crystal properties, which is also in excellent agreement with the theoretical model. Although only a relatively simple configuration was examined, the anisotropic acoustic metamaterial formulation discussed and developed here have the potential for more complicated designs, enabling the construction of effective fluid sound absorbers that have anisotropy in both the material properties and absorption characteristics, as well as the potential for creating enhanced sound absorption performance.

\section{ACKNOWLEDGEMENTS}

This work was supported by the U.S. O ce of Naval Research (Award N000141210216) and by the Spanish Ministerio de Economia y Competitividad (MINECO) under contract No. TEC2010-19751.

${ }^{1}$ J. P. Dowling, "Sonic band structure in fluids with periodic density variations," J. Acoust. Soc. Am. 91, 2539 (1992).

${ }^{2}$ M. Sigalas and M. Economou, "Elastic and acoustic wave band structure," J. Sound. Vib. 158, 377-382 (1992).

${ }^{3}$ J. Sánchez-Pérez, D. Caballero, R. Martínez-Sala, C. Rubio, J. SánchezDehesa, F. Meseguer, J. Llinares, and F. Gálvez, "Sound attenuation by a two-dimensional array of rigid cylinders," Phys. Rev. Lett. 80, 5325-5328 (1998).
${ }^{4}$ W. E. Kock and F. K. Harvey, "Refracting sound waves," J. Acoust. Soc. Am. 21, 471-478 (1949).

${ }^{5}$ F. Cervera, L. Sanchis, J. Sánchez-Dehesa, and F. Cervera, "Refractive acoustic devices for airborne sound," Phys. Rev. Lett. 88, 023902 (2002).

${ }^{6}$ D. Torrent, A. Håkansson, F. Cervera, and J. Sánchez-Dehesa, "Homogenization of two-dimensional clusters of rigid rods in air," Phys. Rev. Lett. 96, 204302 (2006).

${ }^{7}$ D. Torrent and J. Sánchez-Dehesa, "Anisotropic mass density by twodimensional acoustic metamaterials," New. J. Phys. 10, 023004 (2008).

${ }^{8}$ S. A. Cummer, B.-I. Popa, D. Schurig, D. R. Smith, J. B. Pendry, M. Rahm, and A. Starr, "Scattering theory derivation of a 3D acoustic cloaking shell," Phys. Rev. Lett. 74 (2008).

${ }^{9}$ D. Torrent and J. Sánchez-Dehesa, "Acoustic cloaking in two dimensions: a feasible approach," New. J. Phys. 10, 063015 (2008).

${ }^{10}$ J. Li, L. Fok, X. Y. G. Barthal, and X. Zhang, "Experimental demonstration of an acoustic magnifying hyperlens," Nat. mat. 18, 931-934 (2010).

${ }^{11}$ J. B. Pendry and J. Li, "An acoustic metafluid: realizing a broadband acoustic cloak," New J. Phys. 10, 115032 (2008).

${ }^{12}$ B.-I. Popa and S. A. Cummer, "Design and characterization of broadband acoustic composite metamaterials," Phys. Rev. B 80, 174303 (2009).

${ }^{13}$ D. Torrent and J. Sánchez-Dehesa, "Anisotropic mass density by radially periodic fluid structures," Phys. Rev. Lett. 105, 174301 (2010).

${ }^{14}$ L. N. Gumen, J. Arriaga, and A. A. Krokhin, "Metafluid with anisotropic dynamic mass," Low Temp. Phys. 37, 975-978 (2011).

${ }^{15}$ L. Zigoneanu, B.-I. Popa, A. F. Starr, and S. A. Cummer, "Design and measurements of a broadband two-dimensional acoustic metamaterial with anisotropic e ective mass density,” J. Appl. Phys. 109, 054906 (2011).

${ }^{16}$ E. Reyes-Ayona, D. Torrent, and J. Sánchez-Dehesa, "Homogenization theory for periodic distributions of elastic cylinders embedded in a viscous fluid," J. Acoust. Soc. Am. 132, 2896-2908 (2012).

${ }^{17}$ C. J. Naify, C.-M. Chang, G. McKnight, and S. Nutt, "Transmission loss and dynamic response of membrane-type locally resonant acoustic metamaterials," J. Appl. Phys. 108, 114905 (2010).

${ }^{18}$ Z. Yang, H. M. Dai, N. H. Chan, G. C. Ma, and P. Sheng, "Acoustic metamaterial panels for sound attenuation in the 50-1000 Hz regime," Appl. Phys. Lett. 96, 041906 (2010).

${ }^{19}$ C. J. Naify, C.-M. Chang, G. McKnight, F. Schuelen, and S. Nutt, "Membrane-type metamaterials: Transmission loss of multi-celled arrays," J. Appl. Phys. 109, 104902 (2011).

${ }^{20}$ M. I. Hussein and M. J. Frasier, "Metadamping: An emergent phenomenon in dissipative metamaterials," J. Sound Vib. 332, 4767-4774 (2013).

${ }^{21}$ Y. Zhang, J. Wen, H. Zhao, D. Yu, L. Cai, and X. Wen, "Sound insulation property of membrane-type acoustic metamaterials carrying di erent masses at adjacent cells," J. Appl. Phys. 114, 063515 (2013).

${ }^{22}$ J. M. Manimala and C. T. Sun, "Microstructural design studies for locally dissipative acoustic metamaterials," J. Appl. Phys. 115, 023518 (2014).

${ }^{23}$ M. Oudich, X. Zhou, and M. B. Assouar, "General analytical approach for sound transmission loss analysis through a thick metamaterial plate," J. Appl. Phys. 116, 193509 (2014).

${ }^{24}$ J. Christensen, V. Romero-Garcia, R. Pico, A. Cebrecos, F. J. G. de Abajo, N. A. Mortensen, M. Willatzen, and V. J. Sánchez-Morcillo, "Extraordinary absorption of sound in porous lamella-crystals," Sci. Rep. 4, 4674 (2014).

${ }^{25}$ J. Sánchez-Dehesa, V. M. Garcia-Chocano, D. Torrent, F. Cervera, S. Cabrera, and F. Simon, "Noise control by sonic crystal barriers made of recycled materials," J. Acous. Soc. Am. 129, 1173-1183 (2011).

${ }^{26}$ V. M. Garcia-Chocano, S. Cabrera, and J. Sánchez-Dehesa, "Broadband sound absorption by lattices of microperforated cylindrical shells," Appl. Phys. Lett. 101, 184101 (2012).

${ }^{27}$ A. Climente, D. Torrent, and J. Sánchez-Dehesa, "Omnidirectional broadband acoustic absorber based on metamaterials," Appl. Phys. Lett. 100, 144103 (2012).

${ }^{28}$ J.-F. Allard and Y. Champoux, "New empirical equations for sound propagation in rigid frame fibrous materials," J. Acoust. Soc. Am. 91, 3346-3353 (1992).

${ }^{29}$ D. L. Johnson, J. Koplik, and R. Dashen, "Theory of dynamic permeability and tortuosity in fluid-saturated porous media," J. Fluid Mech. 176, 379402 (1987).

${ }^{30}$ V. Tarnow, "Compressibility of air in fibrous materials," J. Acoust. Soc. Am. 99, 3010-3017 (1996). 
${ }^{31}$ C. Peyrega and D. Jeulin, "Estimation of acoustic properties and of the representative volume element of random fibrous media," J. Appl. Phys. 113, 104901 (2013).

${ }^{32}$ C. Perrot, F. Chevillotte, and R. Panneton, "Dynamic viscous permeability of an open-cell aluminum foam: Computations versus experiments," J. Appl. Phys. 103, 024909 (2008).

${ }^{33}$ C. Perrot, F. Chevillotte, and R. Panneton, "Bottom-up approach for microstructure optimization of sound absorbing materials," J. Acoust. Soc. Am. 124, 940-948 (2008).

${ }^{34}$ C. Perrot, F. Chevillotte, M. T. Hoang, G. Bonnet, F.-X. Bécot, L. Gautron, and A. Duval, "Microstructure, transport, and acoustic properties of opencell foam samples: Experiments and three-dimensional numerical simulations," J. Appl. Phys. 111, 014911 (2012).

${ }^{35} \mathrm{~V}$. Tarnow, "Airflow resistivity of models of fibrous acoustic materials," J. Acoust. Soc. Am. 100, 3706-3713 (1996).

${ }^{36} \mathrm{~S}$. Kuwabara, "The forces experienced by randomly distributed parallel circular cylinders or spheres in a viscous flow at small reynolds numbers," J. Phys. Soc. Jpn. 14, 527-532 (1959).

${ }^{37}$ V. Tournat, V. Pagneux, D. Lafarge, and L. Jaouen, "Multiple scattering of acoustic waves and porous absorbing media," Phys. Rev. E 70, 026609 (2004).

${ }^{38}$ P. A. Martin, A. Maurel, and W. J. Parnell, "Estimating the dynamic e ective mass density of random composites," J. Acoust. Soc. Am. 128, 571-577 (2010).

${ }^{39} \mathrm{~K}$. Attenborough, "Acoustical characteristics of rigid fibrous absorbents and granular materials," J. Acoust. Soc. Am. 73, 785-799 (1982).

${ }^{40}$ J. M. Evans and K. Attenborough, "Sound propagation in concentrated emulsions: Comparison of coupled phase model and core-shell model," J.
Acoust. Soc. Am. 112, 1911-1917 (2002).

${ }^{41} \mathrm{R}$. Christensen, Mechanics of Composite Materials (Dover Publications, 2005).

${ }^{42}$ A. Einstein, Investigation of the Brownian movement (Dover, New York, 1956).

${ }^{43}$ M. Schoenberg and P. N. Sen, "Properties of a periodically stratified acoustic half-space and its relation to a biot fluid," J. Acoust. Soc. Am. 73, 61-67 (1983).

${ }^{44}$ W. P. Arnott, H. E. Bass, and R. Raspet, "General formulation of thermoacoustics for stacks having arbitrarily shaped pore cross sections," J. Acoust. Soc. Am. 90, 3228-3237 (1991).

${ }^{45}$ A. D. Pierce, Acoustics: An Introduction To Its Physical Principles and Applications (Acoustical Society of America, 1989).

${ }^{46}$ D. T. Blackstock, Fundamentals of Physical Acoustics, 1st ed. (John Wiley \& Sons, New York, 2000).

${ }^{47}$ V. Fokin, M. Ambati, C. Sun, and X. Zhang, "Method for retrieving e ective properties of locally resonant acoustic metamaterials," Phys. Rev. B 76, 144302 (2007).

${ }^{48}$ A. Baccigalupi, “ADC testing methods,” Measurement 26, 199-205 (1999).

${ }^{49}$ Y. Salissou and R. Panneton, "Wideband characterization of the complex wave number and characteristic impedance of sound absorbers," J. Acoust. Soc. Am. 128, 2868-2876 (2010).

${ }^{50}$ B. H. Song and J. S. Bolton, "A transfer-matrix approach for estimating the characteristic impedance and wave numbers of limp and rigid porous materials,” J. Acoust. Soc. Am. 107, 1131-1152 (2000).

${ }^{51}$ M. D. Guild, V. M. Garcia-Chocano, W. Kan, and J. Sánchez-Dehesa, "Enhanced inertia from lossy e ective fluids using multi-scale sonic crystals," AIP Adv. 4, 124302 (2014). 